\title{
Aa. Vv., Miscellanea in memoriam Pierre Cockshaw (1938-2008), Aspects de la vie culturelle dans les Pays-Bas Méridionaux (XIV ${ }^{e}$ XVIII ${ }^{e}$ siècle)
}

\section{Maria Colombo Timelli}

\section{(2) OpenEdition}

\section{Journals}

\section{Édition électronique}

URL : http://journals.openedition.org/studifrancesi/5550

DOI : ERREUR PDO dans /localdata/www-bin/Core/Core/Db/Db.class.php L.34 : SQLSTATE[HY000]

[2006] MySQL server has gone away

ISSN : 2427-5856

\section{Éditeur}

Rosenberg \& Sellier

Édition imprimée

Date de publication : 1 septembre 2011

Pagination : 391-392

ISSN : 0039-2944

\section{Référence électronique}

Maria Colombo Timelli, «Aa. Vv., Miscellanea in memoriam Pierre Cockshaw (1938-2008), Aspects de la vie culturelle dans les Pays-Bas Méridionaux (xıve-xvIII siècle)», Studi Francesi [En ligne], 164 (LV | II) | 2011, mis en ligne le 30 novembre 2015, consulté le 08 janvier 2021. URL : http:// journals.openedition.org/studifrancesi/5550 ; DOI : https://doi.org/ERREUR PDO dans /localdata/ www-bin/Core/Core/Db/Db.class.php L.34 : SQLSTATE[HY000] [2006] MySQL server has gone away

Ce document a été généré automatiquement le 8 janvier 2021.

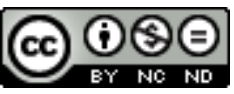

Studi Francesi è distribuita con Licenza Creative Commons Attribuzione - Non commerciale - Non opere derivate 4.0 Internazionale. 


\title{
Aa. Vv., Miscellanea in memoriam Pierre Cockshaw (1938-2008), Aspects de la vie culturelle dans les Pays-Bas Méridionaux (XIV ${ }^{e}-\mathrm{XVII}{ }^{e}$ siècle)
}

\author{
Maria Colombo Timelli
}

\section{RÉFÉRENCE}

Miscellanea in memoriam Pierre Cockshaw (1938-2008), Aspects de la vie culturelle dans les PaysBas Méridionaux (XIV ${ }^{\mathrm{e}}$ XVIII ${ }^{\mathrm{e}}$ siècle), Bruxelles, Archives et Bibliothèques de Belgique, 2009, 2 volumes.

1 Conçu d'abord en l'honneur de Pierre Cockshaw, Conservateur en chef de la Bibliothèque royale de Belgique (les contributions datent des années 2004-2005), ce recueil in memoriam réunit un certain nombre d'articles intéressant notre «Rassegna».

2 Renaud ADAM, La circulation des incunables à Louvain au XVe siècle: étude sur la production du relieur Ludovicus Ravescot, pp.1-21. La présence de l'Université et de nombreuses institutions ecclésiastiques ont certes contribué à l'implantation précoce d'ateliers typographiques à Louvain (dès 1473). R.A. analyse ici les livres reliés par L. Ravescot, qui fut successivement enlumineur, libraire, imprimeur, actif entre 1475 et 1487. Les 58 reliures conservées de lui renferment pour la plupart des incunables datés du dernier tiers $\mathrm{du} \mathrm{XV}^{\mathrm{e}}$ siècle, provenant surtout de Cologne et de Venise; les propriétaires en étaient tant des religieux que des universitaires. Parmi ces œuvres, toutes en latin, priment les écrits religieux et juridiques, mais la présence du Compendium elegantiarum Laurentii Vallensis de Bonus Accursius témoigne aussi du rôle des imprimeurs louvanistes dans la diffusion de l'humanisme italien.

3 Georges colin (Reliures en portefeuille confectionnées au prieuré de Groenendael, pp. 117-125) traite d'un type particulier de reliures, souples à rabat, réalisées à Groenendaal, non 
loin de Bruxelles, entre la fin du $\mathrm{XV}^{\mathrm{e}}$ et le début $\mathrm{du} \mathrm{XVI}^{\mathrm{e}}$ siècle (Bruxelles, KBR, mss 2137-2138, 11778-11782, 11733-11739; Paris, Mazarine, ms 450; Oxford, Bodleian Library, Depot Ehrman 32.11).

Thérèse DE HEMPTINNE (Thérèse, Ferrand, Isabelle et leurs époux. Les alliances matrimoniales portugaises des comtes et comtesses de Flandre, 1184-1526, pp. 127-136). Parmi les comtes et comtesses de Flandres qui épousèrent au Moyen Âge des princes portugais, se signale Philippe le Bon; Isabelle du Portugal, qu'il épousa en troisièmes noces en 1430, représentait de plusieurs points de vue la duchesse idéale: elle apportait une dot importante, put jouer le rôle de médiatrice avec l'Angleterre, et partageait avec son mari l'intérêt pour la défense de la foi chrétienne et donc pour le projet de croisade; enfin elle lui donna un fils, Charles, destiné à régner après Philippe. Loin d'oublier ses origines, elle sut s'entourer de marchands, artisans, artistes et savants portugais qui enrichirent la cour de Bourgogne d'apports divers.

5 Geneviève HASENOHR (Un faux Pierre de Luxembourg, un vrai Arnoul de Bohéries et un Isidore travesti dans la bibliothèque de Marguerite d'York, pp. 175-193) étudie les sources du Traité qui montre comment on doit ordonner tout son temps à servir Dieu et à l'aimer avec ferveur, dont un manuscrit, confectionné par David Aubert, a appartenu à Marguerite d'York (Oxford, Bodleian Library, Douce 365); elle les identifie dans le Speculum monachorum (xII ${ }^{\mathrm{e}}$ siècle) pour la première partie, et dans une série des Synonyma d'Isidore de Séville pour la deuxième.

6 Frédérique JoHan (Les artisans du livre sous les gouvernements de Philippe le Bon et de Charles le Téméraire: en marge d'un projet d'édition de sources, pp. 223-241) aborde la question des mécanismes de confection et de payement des livres manuscrits à la cour de Bourgogne, objet d'une base de données pour la période 1419-1477. Sont ainsi classées 10 prestations: translation du texte, copie, enluminure, reliure, restauration, achat, convoyage, don, prestations exceptionnelles, sous-traitance. Les actes révèlent l'importance de cette dernière pratique, assumée par des enlumineurs, écrivains, copistes ou traducteurs qui devenaient de véritables gestionnaires/entrepreneurs polyvalents. Quant à la rémunération, elle se faisait normalement à la pièce, mais elle pouvait se transformer en une pension annuelle.

7 Jacqueline LECLERCQ-MARX (La représentation des dieux antiques dans le premier volume des Chroniques de Hainaut. L'image, le texte, le contexte et la postérité, pp. 243-279) étudie les enluminures figurant aux ff. $173 \mathrm{v}-175 \mathrm{v}$ du célèbre manuscrit 9242 de la KBR: exceptionnelles à plusieurs égards, elles représentent neuf dieux antiques selon un mode qui fusionne la tradition des idoles médiévales et celle des dieux de la Renaissance; elles confirment en même temps le goût de Philippe le Bon pour la mythologie antique et le caractère transitoire d'une production destinée à être plus strictement codifiée vers la fin $\mathrm{du} \mathrm{Xv}^{\mathrm{e}}$ siècle. En annexe, les passages des Chroniques concernant les dieux (Baal, Bacchus, Saturne, Jupiter, Mars, Apollon, Vénus, Mercure, Diane) et la reproduction de 18 enluminures, dont 5 tirées du ms. de Bruxelles.

Claudine LEMAIRE (Jean Molinet, grand rhétoricien, et la devise de Baudouin de Lannoy, pp. 281-289) explore les rapports qui s'instaurèrent entre l'indiciaire bourguignon et Baudouin de Lannoy, ce que prouverait entre autres la devise Ote lannoy de Baudechon (= 'écarte les soucis du petit baudet', Baudechon étant le diminutif tant de Baudouin que de baudet), dont Molinet serait l'auteur. 
9 Jacques Charles LEMAIRE (Un poème inédit sur les saisons: "L'histoire d'ivier et de prinztampz", pp. 291-305) donne l'édition d'un poème de 256 vers, contenu dans le ms. Bruxelles, KBR IV 541, dont il souligne les caractères originaux: il s'agit d'abord d'un texte narratif, qui alterne parties descriptives et parties dialoguées, dont le récit est précisément inscrit en Picardie. La langue (on soulignera la présence de 5 hapax lexicaux) en situerait la composition au $\mathrm{XVI}^{\mathrm{e}}$ siècle.

10 Françoise MURET et Ludovic NYS (Les peintures de la Salle-le-Comte de Valenciennes, pp. 323-374) étudient le décor mural de ce complexe d'édifices, où étaient représentés quatre sujets bien connus des médiévistes: le Pas Saladin, la Fontaine de jouvence, le Jeu d'échecs et le Mercier aux singes. La question du concepteur de ce projet demeure ouverte, mais il n'est pas invraisemblable de penser à une intervention de Jean Froissart. Les chapitres des comptes de Valenciennes relatifs à ce décor figurent en annexe.

11 L'article de Werner PARAVICINI (Secrétaires criminels. La conscience des clercs au service de l'État au XVe siècle, pp. 375-412) concerne une question peu étudiée, à savoir la situation des clercs devenus «criminels» et récompensés par des dons ou des offices da la part du duc. En annexe, on trouvera un dossier de dix documents datés 1386-1440 et une riche bibliographie.

12 Jacques раvіот (Mentions de livres, d'auteurs, de copistes, d'enlumineurs, de miniaturistes ("historieurs") et de libraires dans les comptes généraux du duc de Bourgogne Philippe le Bon, pp. 413-446) élargit l'enquête menée par P. Cockshaw («Scriptorium», XXIII, 1969) au règne de Philippe le Bon. Il publie ainsi 165 documents d'archives pour la période 1419-1467 du plus grand intérêt pour les spécialistes de l'histoire culturelle du duché de Bourgogne, où l'on relève, à côté de noms moins connus, la mention de personnages célèbres: des peintres (Jan Van Eyck), enlumineurs (Dreux Jean, Loyset Liédet, Jean le Tavernier), translateurs (Jean Wauquelin, Jean Miélot), escripvains (Jean Miélot, Colard Mansion), croniqueurs (Enguerran de Monstrelet, Edmond de Dynter, Georges Chastelain), ainsi que de grands seigneurs de la cour, tels Jean de Croÿ, ayant avancé des sommes pour le compte du duc à Jacotin du Bois, Jean Wauquelin, Jacotin Pilavaine (notice 138), ou «Jehan Arnoulphin, marchant de Lucques residant a Bruges» (notice 121), bien connu grâce au portrait de Jan Van Eyck lui-même.

13 Anne VAN BUREN (The Hard Life of a Fifteenth-Century Artist: Colard le Voleur, pp. 497-513) introduit et commente quatre documents concernant les travaux effectués par Colard le Voleur lors des rénovations du château de Hesdin sous Philippe le Bon; la supplique que cet artiste, "pauvre homme (et non pas: honnne, sic!) chargié de femme et de pluiseurs petis enfans», adressa au Duc en 1435 est un témoignage exceptionnel dans la Bourgogne $\mathrm{du} \mathrm{xv}^{\mathrm{e}}$ siècle. On regrette d'autant plus les nombreuses fautes de transcription, qui faussent le sens de cette pièce (p. 511, «pour vous faire plaisir», et non pas vois; p. 512, "et se luy a convenu trouver bos et autre matieres...», et non pas seluy; surtout, «lau» ('là') et non pas l'au (trois fois dans la même p. 512).

Baudouin VAN DEN ABEELE (Jean Molinet et Guillebert de Lannoy: un nouveau témoin et un manuscrit retrouvé, pp. 515-525) analyse de façon approfondie le ms. II 6978 de la KBR, dont l'intérêt ne fait pas de doute: daté de 1482, il a appartenu à Charles de Crö̈, prince de Chimay. Il contient une quinzaine de titres, parmi lesquels il faut signaler quatre œuvres de Jean Molinet (Trosne d'honneur, Epitaphe de Philippe le Bon, Naufrage de la Pucelle, Ressource du petit peuple), une copie - incomplète et irrégulière - des Voyages et Ambassades de Guillebert de Lannoy, la version longue du Bestiaire de Pierre de 
Beauvais, outre des poèmes plus brefs et moins connus ou à caractère circonstanciel. Recueil didactique et moral, ce manuscrit pourrait avoir été composé à l'initiative de Philippe de Croÿ.

15 Christiane VAN DEN BERGEN-PANTENS (Portraits troyens et héraldique imaginaire. Les exemples d'Hector et de Penthésilée, pp. 527-537) suit l'histoire des armoiries attribuées au héros troyen et à la reine des Amazones dans la tradition médiévale, de Benoît de SainteMaure aux XIV ${ }^{e}-X^{e}$ siècles (armoriaux et textes littéraires, notamment pour le cycle des Neuf Preux et des Neuf Preuses). 